\title{
(Re-)Produktion von Differenzen in unterrichtlichen Praktiken
}

\section{Tanja Sturm}

In diesem Aufsatz wird entlang eines wissenssoziologischen Zugangs der Frage nachgegangen, wie Lehrpersonen in unterrichtlichen Praktiken Differenzen zwischen Schüler/innen herstellen und bearbeiten. Anhand von empirischem Material aus Gruppendiskussionen werden zwei unterschiedliche Typen illustriert: ein an einer totalen Norm orientierter und ein situativ-angleichend ausgerichteter Typ. Beide Formen behindern und eröffnen Lernmöglichkeiten im Unterricht. Sie liefern weiter Hinweise darauf, dass formale schulische Regelungen in den unterrichtlichen Praktiken von Lehrkräften hohe Bedeutung haben. Bei der Gestaltung einer inklusiven Unterrichts- und Schulpraxis sind diese Orientierungspunkte zu berïcksichtigen.

\section{Heterogenität und Differenzen}

Die Frage nach schulischem und unterrichtlichem Umgang mit Heterogenität ist seit etwa 20 Jahren eine zentrale innerhalb des erziehungswissenschaftlichen Diskurses (z.B. Budde, 2012). Sie steht in enger Verbindung mit Kritik am aktuellen - Benachteiligungen hervorbringenden - Umgang durch Schule und Unterricht. Wie v.a. gross angelegte Studien empirisch belegen (für $\mathrm{CH}$ : z.B. Zahner Rossier \& Holzer, 2007), finden nach wie vor systematische Benachteiligungen für Angehörige sozialer Gruppen wie z.B. Schüler/innen aus sozio-ökonomisch benachteiligten Milieus statt. Die Realisierung von Chancengleichheit, unabhängig vom sozialen und kulturellen Hintergrund der Schüler/innen erfolgt nicht zufriedenstellend. Folglich berührt die Diskussion um Heterogenität auch die Legitimität der Schule, die niemanden diskriminieren darf. Die Situation zu verändern, ist breiter Konsens in der erziehungswissenschaftlichen Theoriebildung (z.B. Prengel, 2006) und spitzt sich als organisatorische und professionelle Herausforderung in der Frage nach der Gestaltung einer integrationsfähigen Schule zu (Bless \& Kronig, 1999). Barrieren und Behinderungen in schulischen Lehr-Lernprozessen, insbesondere jene, die systematisch zur 
Benachteiligung sozialer Gruppen führen, zu überwinden, ist das Ziel der Inklusionspädagogik. Neben schulstrukturellen Änderungen werden Praktiken von Lehrpersonen als zentraler Ansatzpunkt hierfür gesehen; Kenntnisse darüber, wie Differenzen unterrichtlich hergestellt und zu Barrieren in Lehr-Lernprozessen werden, liegen nur in Ansätzen vor (Ainscow, 2008).

Vor dem skizzierten Hintergrund wird die Weiterentwicklung von Unterricht gefordert, häufig, ohne die spezifischen Bedingungen in der Organisation Schule (Sturm, 2010, 146 f.) und die Einbindung schulischer Akteure/innen in die (Re-)Produktion von Differenz zu reflektieren (Mecheril \& Plösser, 2009, 200 f.). Dieses Desiderat soll mithilfe einer praxeologisch-wissenssoziologischen Perspektive aufgegriffen werden, indem gefragt wird, wie in unterrichtlichen Praktiken von Lehrkräften Differenzen bearbeitet undloder hergestellt werden. Die mikroanalytische Betrachtung nimmt jene Praktiken und Prozesse in den Blick, mit denen soziale Realität - hier die Konstruktion und Bearbeitung von Differenzen - vorgenommen werden. Im Gegensatz zu anderen konstruktivistischen Perspektiven wird der Kontext der Organisation Schule mit ihren formalen Regeln, die sie an ihre Mitglieder stellt (Nohl, 2007b, S. 69 f.), nicht ausgeblendet. Heterogenität respektive Differenzen werden als Ausdruck unterschiedlicher alltäglicher Lebenspraxen verstanden. Innerhalb von Organisationen, wie der Schule, entwickeln sich spezifische Milieus. Im Kontext von Schule und Unterricht sind die professionellen Milieus von Lehrpersonen, denen sich dieser Aufsatz widmet, von jenen der Schüler/innen zu unterscheiden. Differenzkonstruktionen erfolgen in zwei nicht voneinander losgelösten Modi: explizit, d.h. begrifflich, wie z.B. in Form verbaler Äusserungen, und implizit, also in körperlichen und sprachlichen Praktiken (Bohnsack \& Nohl, 2001, S. 22). Dieser Aufsatz fokussiert die letztgenannten Differenzkonstruktionen und ihre Bearbeitungsformen in professionellen Milieus von Lehrkräften. Um diesem Interesse nachzugehen, werden Ergebnisse aus der Untersuchung Differenzkonstruktionen in unterrichtlichen Praktiken (z.B. Sturm, 2012) illustrierend herangezogen. Im Anschluss an eine Skizzierung der theoretischen und methodologischen Vorgehensweise der Studie (Abschnitt 2) werden zwei Typen der Herstellung und Bearbeitung von Differenzen illustriert und miteinander verglichen (Abschnitt 3). Abschliessend werden die Ergebnisse mit den einleitenden theoretischen Überlegungen in Beziehung gesetzt und diskutiert (Abschnitt 4).

\section{Differenzkonstruktionen in unterrichtlichen Orientierungen}

Die Studie Differenzkonstruktionen in unterrichtlichen Praktiken folgt den theoretischen Überlegungen der praxeologischen Wissenssoziologie, die methodologisch mit der dokumentarischen Methode bearbeitet werden. Heterogenität wird innerhalb des Ansatzes als Ausdruck der Vielfalt des menschlichen 
Alltags in pluralen Gesellschaften verstanden, die zu unterschiedlichen Praxen der Lebensführung führen, zu sogenannten Milieus (Nohl, 2010, S. 145 ff.). Differenzen beschreiben Unterschiede zwischen milieuspezifischen Praxen, während Gemeinsamkeiten zwischen oder innerhalb von Milieus als Konjunktionen gefasst werden. Differenzkonstruktionen aus wissenssoziologisch-praxeologischer Perspektive heraus zu betrachten, erfordert die Unterscheidung von zwei Wissensformen: dem explizit-kommunikativen Wissen und dem konjunktiven Erfahrungswissen. Letzteres ist perspektivisch gebunden und resultiert aus existenziellen sozialen und materiellen Beziehungen. So liegt die Rolle, Schülerin zu sein, explizit vor und ist zugleich mit konkreten sozialen Erfahrungen mit den Mitschüler/innen, den konkreten Schulgebäuden und Pausenhöfen, den Lehrpersonen, den Unterrichtsfächern u.v.m. verknüpft. Dieses «atheoretische» Wissen wird in gemeinsamen Erfahrungen mit und gegenüber anderen $/ \mathrm{m}$ entwickelt und steht, anders als das explizit-kommunikative, i.d.R. nicht begrifflich und reflexiv zur Verfügung. Menschen, die sich in erfahrungsgebundener, vergleichbarer, nicht notwendigerweise gleichartiger Art und Weise auf Situationen und/oder Gegenstände beziehen, gehören einem Milieu an. Soziale Milieus sind nicht ein-, sondern mehrdimensional konzipiert, d.h. einzelne Akteure/innen gehören gleichzeitig mehreren, einander überlappenden Erfahrungsräumen an, so z.B. einem geschlechts- und einem professionellen Milieu. Menschliche Praktiken orientieren sich wesentlich am Erfahrungswissen, das einen zentralen Bestandteil der Erfahrungsgesamtheit ausmacht (Mannheim, 1980, S. 212). Das schulische Organisationsmilieu wird hier in der Dimension des «Unterrichtsmilieus» (Wagner-Willi \& Sturm, 2012) betrachtet. Aufseiten der Lehrpersonen umfasst dieses neben dem sozialen Milieu, dem sie angehören, ihre pädagogisch-professionelle, durch die Organisation geprägte Rollenzugehörigkeit ebenso wie den Unterrichtsgegenstand, die unterrichtliche Sozialform sowie die soziale Milieuzugehörigkeit der Schüler/innen. In der Unterrichtspraxis sind diese Aspekte im Sinne kommunikativer und reflexiver Wissensformen sowie der mit ihnen verbundenen konjunktiven und kommunikativen Beziehungen miteinander sprachlich wie körperlich verwoben. Die Rolle der Lehrpersonen ist durch die Zielsetzung gekennzeichnet, Bildungs- und Erziehungsprozesse aufseiten der Schüler/innen zu initiieren (Nohl, 2007b, S. 70).

Das professionelle Erfahrungswissen und die handlungsleitenden Orientierungen der Differenzkonstruktionen von Lehrpersonen sind "[...] nicht Gegenstand expliziter, d.h. theoretisch-reflexiver Darstellungen. Sie begegnet uns in impliziter oder atheoretischer Weise d.h. in Form von Beschreibungen und Erzählung der Alltagspraxis [...]» (Bohnsack \& Nohl, 2001, S. 22, Herv. im Original). Empirisch erfolgt die Rekonstruktion des unterrichtlichen Erfahrungswissens der Lehrpersonen mithilfe der dokumentarischen Methode, die an die wissenssoziologische Perspektive Mannheims (1980) anknüpft und von Bohnsack (2010) massgeblich weiterentwickelt wurde. Die Unterscheidung zwischen explizit-kommunikativem Wissen und dem konjunktiven Erfahrungs- 
wissen, d.h. zwischen immanentem und dokumentarischem Gehalt ist fundamental für die Analyseeinstellung der dokumentarischen Methode (Przyborski \& Wohlrab-Sahr, 2009, S. 277). Das handlungsleitende Orientierungswissen wird in Erzählungen und Beschreibungen von Akteuren/innen mit homologem Erfahrungswissen reaktualisiert und in einem mehrschrittigen Interpretationsprozess rekonstruiert. Während mit der formulierenden Interpretation Themen und Inhalte des Gesprächs textnah herausgearbeitet werden, erfolgt in der darauffolgenden reflektierenden Interpretation die Rekonstruktion der Orientierungsrahmen, in denen Themen und Inhalte zwischen Beteiligten verhandelt und gemeinsame Bezugspunkte gesetzt werden. Der mit dem Ziel der Typisierung vorgenommene Vergleich von Orientierungsrahmen unterschiedlicher Gruppendiskussionen stellt zugleich einen Generalisierungsschritt dar und erhöht die Validität der Rekonstruktion (Bohnsack, 2010, S. 134 ff.). Die im Rahmen der Untersuchung mit einem Diktiergerät aufgenommenen Gespräche wurden in geschriebene Text übersetzt, die die Grundlage der Auswertung darstellen.

\section{Totale und fachbezogene Konstruktion von Differenzen}

In diesem Abschnitt sollen Teilergebnisse der Untersuchung Differenzkonstruktionen in unterrichtlichen Praktiken herangezogen werden, um nachvollziehbar zu machen, wie Differenzen im Unterricht hergestellt und bearbeitet werden. Der Studie liegen Gruppendiskussionen, die an unterschiedlichen deutschen und österreichischen Schultypen und in unterschiedlichen Sozialräumen geführt wurden, zugrunde. Entsprechend den Ergebnissen der dokumentarischen Interpretation gehen mit den Orientierungen der Lehrpersonen grundlegende Erwartungen an die Schülerrolle und Vorstellungen unterrichtlicher Bearbeitung einher. Hinsichtlich der Orientierungsrahmen der Lehrpersonen lassen sich vier Formen der Differenzkonstruktion und -bearbeitung unterscheiden: totale Zuschreibung und Homogenität erreichen (Typ 1), totale Zuschreibung und Heterogenität akzeptieren (Typ 2), partielle Zuschreibung und Homogenität erreichen (Тyp 3) und partielle Zuschreibung und Heterogenität akzeptieren (Typ 4). Zwei der insgesamt vier Typen sollen exemplarisch illustriert werden: Typ 2 und Typ 4. Sie unterscheiden sich in beiden Dimensionen und stehen somit in maximalem Kontrast zueinander. Illustrierend wird je eine Passage einer Gruppendiskussion in Form eines Transkripts vorgestellt, dann die rekonstruierten Orientierungsrahmen, um sie abschliessend miteinander zu vergleichen.

\section{Typ Totale schulsystembezogene Konstruktion von Differenzen}

An der Gruppendiskussion sind fünf Lehrerinnen ${ }^{1}$ einer städtischen, integrativen Grundschule einer westdeutschen Grossstadt beteiligt. Unmittelbar vor 
der Passage verhandelt die Gruppe Unklarheiten bei der Vergabe von Noten für die Integrationskinder. Es handelt sich hierbei um Kinder, die sonderpädagogischen Förderbedarf in den Bereichen Lernen und/oder Sprache haben und die integrativ 2 in der Grundschule beschult werden.

\begin{tabular}{|c|c|c|}
\hline 962 & Cf: & Also ich glaube auch das sind wirklich die Kinder die haben \\
\hline 963 & & also ich mein ich will jetzt nicht sagen die haben es gut in \\
\hline 964 & & der Richtung aber die sind nicht so für uns eh glaube hier als \\
\hline 965 & & Grundschullehrer die so problematisch sind eh was wir jetzt \\
\hline 966 & & eben hier beschrieben haben ich meine jetzt nicht die das \\
\hline 967 & & persönliche Kind sondern wie man damit umgeht denn die haben \\
\hline 968 & & ja son Sonderstatus die sind ja ausgewiesene Sonderschüler und \\
\hline 969 & & alles gilt irgendwie etwas anders aber bei den restlichen \\
\hline 970 & & Kindern haben wir ja auch Kinder dabei die ganz sch-viele \\
\hline 971 & & Schwierigkeiten mit $\mathrm{m}$ Lernen haben und dann lassen wir sie \\
\hline 972 & & manchmal überprüfen weil ich denke das Kind kann ja gar nichts \\
\hline 973 & & und dann wird eben festgestellt hat nicht diesen Sonderschul \\
\hline 974 & & eh Bedarf sondern es ist ein Regelkind nicht hochbegabt aber $\mathrm{n}$ \\
\hline 975 & & Regelkind und die die Schwierigkeiten liegen in ganz vielen \\
\hline 976 & & Bereichen die ehm in der gesamten Entwicklung dazu geführt \\
\hline 977 & & haben bis hin zu stark auffälliges Verhalten dass das Kind gar \\
\hline 978 & & nicht in der Lage ist zu lernen weil es nicht zur Ruhe kommt \\
\hline 979 & & weil es sich nicht konzentrieren kann und und und und die eh \\
\hline 980 & & find sind also für mich diejenigen die mir Schwierigkeiten \\
\hline 981 & & machen in den Ansprüchen und in der Bewertung denn auch wenn \\
\hline 982 & & ich Lernstand nehme wo Frau eh wo Ef eben von gesprochen hat \\
\hline 983 & & diese Lernstandserhebungen dann nehmen die I-Kinder nicht teil \\
\hline 984 & Bf: & Und wenn sie Noten bekämen bekämen sie sowieso andere $[\ldots]$ \\
\hline 988 & Df: & Also die ärmsten Würmer sind eigentlich die finde ich die so \\
\hline 989 & & gerade an der Grenze \\
\hline 990 & Cf: & $\mathrm{Ja}$ \\
\hline 991 & Df: & vorbeischrammen die dann irgendwie $n$ IQ \\
\hline 992 & & von hundert haben sag ich jetzt mal oder neunzig \\
\hline 993 & Cf: & Ja drunter meistens \\
\hline 994 & Df: & Meistens noch drunter irgendwie bei neunzig wo eben definitiv \\
\hline 995 & & keine sonderpädagogische Förderung irgendwie \\
\hline 996 & Bf: & Förderbedarf schon aber kein sonderpädagogischer \\
\hline 997 & Cf: & ja genau so \\
\hline
\end{tabular}

Inhaltlich geht es in diesem Teil der Passage um drei unterschiedliche Gruppen von Schüler/innen: die «I-Kinder», die so genannten «hochbegabten Regelkinder» und jene, die zwar Regelkinder sind, aber keinen Anspruch auf sonderpädagogische Unterstützung erhalten (962-997). Die letztgenannte Kindergruppe wird zwar den Regelkindern zugeschrieben, unterscheidet sich jedoch von ihnen, da die Schüler/innen «nicht hochbegabt» sind und ihr Intelligenzquotient nicht so niedrig ist, dass sie zur Gruppe der Integrationskinder gezählt werden (974-983). Letztere Gruppe zeichnet sich durch besondere Behandlung wie z.B., nicht an Lernstandsvergleichen teilnehmen zu müssen, aus. Der Intelligenzquotient als scheinbar objektive Differenzkategorie wird in seiner Bedeutung für die Lehrpersonen deutlich, da er als Einteilungskriterium zugrunde gelegt wird 
(992). Die Gruppe greift eine schulsystembezogene Differenzdimension auf, die handlungsleitend für ihre unterrichtlichen Angebote sind. Die Differenzkonstruktion ist wesentlich durch schulstrukturelle Kategorien gerahmt.

Die Lehrerinnen beschreiben unterschiedliche Kindergruppen in der Passage und nehmen gleichzeitig Bezug zur unterrichtlich-schulischen bzw. allgemein unterstützenden Zuständigkeit für diese Kinder durch Lehrpersonen; v.a. schulisch-institutionelle Fördermassnahmen, die ausserhalb ihres Zuständigkeitsbereichs liegen, werden genannt. Der Gesprächsfokus liegt auf der Gruppe nicht-hochbegabter Regelkinder, die nach Ansicht der Lehrerinnen viele Probleme und Schwierigkeiten haben (962; 970), ohne Anspruch auf sonderpädagogische Unterstützung (995). Der sonderpädagogische Förderbedarf wird als schulisch-administrative Kategorie formuliert, der jenseits eigener Beobachtungen angesiedelt ist, da er in einem formalen Überprüfungsverfahren ermittelt wird. Die eigenen Beobachtungen der Lehrerinnen und die formal ermittelten Ergebnisse widersprechen sich. In diesem Dilemma scheint das «Schicksal» der Kinder sich schulisch zu entfalten.

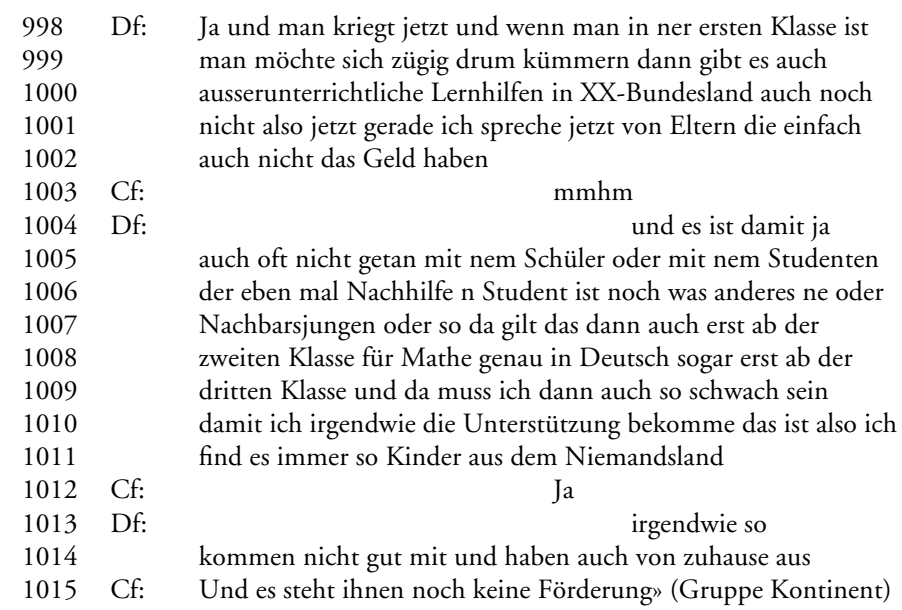

Die Gruppe diskutiert ihre eigenen Schwierigkeiten mit den Kindern, die weder sonderpädagogischen Förderbedarf haben, noch «hochbegabte Regelkinder» sind (980). Die potenzielle Unterstützungsmöglichkeiten für diese Kinder sehen sie in ausserunterrichtlichen Förderangeboten (998). Die Beantragung dieser unterstützen sie ebenso wie eine Überprüfung der Kinder auf sonderpädagogischen Förderbedarf. Neben den ausserschulischen Hilfen sehen sie die Eltern der Kinder als eine weitere Gruppe, die Verantwortung für die nachholende Unterstützung unterrichtlicher Lernprozesse tragen könnte. Im Fall der beschriebenen Kinder fallen diese jedoch aus (1000).

Der positive Horizont, auf den die Diskussion zustrebt, markiert die Schwierigkeiten der Lehrerinnen im Unterricht, für erfolgreiche Lernprozesse der Schüler/ 
innen zu sorgen. Sie verweisen darauf, dass die metaphorisch mit «Kinder aus dem Niemandsland» (1011) umschriebene Gruppe keine besondere Förderung erhält, da keine Ressource für ausserschulische Hilfen bereitgestellt wird. Die Orientierung verweist auf eine Ressourcenproblematik, die zu überwinden wäre, um den Schüler/innen die benötigte Unterstützung zu gewähren. Individuelle Förderung als Konzept besteht für die Lehrerinnen offenbar nur im Rahmen der organisatorischen Kategorie des sonderpädagogischen Förderbedarfs. Sie fühlen sich für die Förderung der «Kinder aus dem Niemandsland» im Regelunterricht nicht zuständig. Misserfolge werden von den Lehrpersonen über die Gruppe, die «nicht gut mitkommt», zugespitzt geäussert. So wird die Vorstellung von normalem Lernen, dem Mitkommen im Unterricht deutlich. Die sogenannten I-Kinder sind von solchen Leistungserwartungen befreit, ihnen gegenüber wird der Anspruch nicht erhoben, da davon ausgegangen wird, dass sie die Ziele nicht erreichen können.

Die Orientierung innerhalb der Passage deutet ein Verständnis von persönlichem Pech an, das die «Kinder aus dem Niemandsland» zu erdulden haben. Die Lehrerinnen empfinden zwar Empathie für sie, jedoch keine Zuständigkeit für ihre Lernprozesse respektive die Gestaltung eines adäquaten unterrichtlichen Angebots. Die Situation der Kinder kann aus der Sicht der Lehrerinnen nur durch ausserschulische Unterstützung, für die jedoch kein rechtlicher Anspruch besteht, ausgeglichen werden. Auf beides haben die Lehrerinnen keinen Einfluss. Vielmehr verstehen sie unterrichtliche Lernprozesse, unterrichtliches Mitkommen, als gleichschrittig und an einem normierten Tempo ausgerichtet, und argumentieren in schulrechtlichen Kategorien, die im Modus totaler Zuschreibung erfolgt. Die Gruppe Kontinent sieht die Zugehörigkeit zu zwei - sich diametral gegenüberstehenden - und prinzipiell ausschliessenden Gruppen vor (Garfinkel, 1956, S. 422), denen sich aus ihrer Sicht alle Schüler/ innen zuordnen lassen; auch wenn die schulische Realität etwas anderes vorsieht. Ihre unterrichtlichen Praxen sind hingegen auf diese zwei Gruppen ausgerichtet, die von unterschiedlichen Professionsgruppen adressiert werden. Ihre Vorstellungen spiegeln die Strukturnotwendigkeit des Sortierens und der Zuordnung im deutschen Schulsystem wider. Die darin enthaltene Erwartung drückt eine Passung zwischen familiärer Unterstützung und schulischen Lernmöglichkeiten aus. Dort, wo Eltern ihren Kindern keine (schuladäquate) Unterstützung bieten bzw. wenn diese nicht zur schulischen Erwartung passt, sollte dies gesellschaftlich durch ausserschulische Massnahmen kompensiert werden. Darin ist die Orientierung enthalten, dass die Kinder zur Schule - und den in ihr durchgeführten Lernstandserhebungen - passen sollen, und eine Vorstellung über Schüler/innen formuliert. Die Differenz, die für die Lehrkräfte leitend in der Betrachtung ihrer Lerngruppen ist, liegt in der Relation der Schüler/innen zu dieser Erwartung und der je individuellen Möglichkeit der Kinder, sie zu erreichen. Die Zuschreibung wird allumfassend verstanden und entsprechend auch die Einteilung der Schüler/ innen. Die Differenzen werden von den Lehrkräften im Unterricht ignoriert. Sie 
erleben sich selbst nicht als aktive Akteurinnen in der Bearbeitung der Heterogenität. Diese verorten sie ausserhalb des Unterrichts und der Schule und konzipieren sie als Kompensation von Defiziten. Hierin wird ein naives Verständnis von Differenz erkennbar, in dem diese als quasi natürlich gegeben konzipiert wird (Mecheril \& Plösser, 2009, S. 200).

Die Bewältigung der Schwierigkeiten, die sie bei den Kindern beobachten und die sich in der Beurteilungsproblematik ausserschulischer Lernhilfen zuspitzen, erleben die Lehrpersonen als eine Überlastung, der durch entlastende Lernzielbefreiung (bei den I-Kindern) oder durch Delegation der Verantwortung an ausserschulische Einrichtungen zu begegnen wäre. Konträr dazu stünde der - unausgesprochene - negative Horizont, mittels eigener unterrichtlicher Praktiken die Lernprozesse aller zu unterstützen. Die realisierten Praktiken sind wesentlich konträr hierzu ausgerichtet, wozu gehört, dass einige Kinder «ins Niemandsland» «verloren gehen».

\section{Typ Partiale, fachbezogene Konstruktion von Differenzen}

Die Gruppe Gipfel, die den vierten Typ repräsentiert, setzt sich aus drei Lehrern und einer Lehrerin zusammen, die v.a. Naturwissenschaften im gymnasialen Zweig einer kooperativen Gesamtschule einer westdeutschen Kleinstadt unterrichten. Die ausgewählte Passage entstammt dem ersten Drittel des Gesprächs und der unmittelbar die Bitte der Diskussionsleiterin vorausgeht, die aufgeworfenen Unterschiede zwischen Schüler/innen anhand unterrichtlicher Beispiele zu konkretisieren.

\begin{tabular}{|c|c|c|}
\hline 593 & Cf: & Also ich hatte das in Chemie zum Beispiel mal in nem \\
\hline 594 & & Leistungskurs vor ein paar Jahren da hatte eine Hälfte da war \\
\hline 95 & & das auch noch mit der mit der Schwerpunktsetzung die eine \\
\hline 596 & & Hälfte hatte Matheleistung und die andere Hälfte ja die hatte \\
\hline 597 & & was anderes und ehm denn ging es darum::: zum Beispiel um \\
\hline 98 & & Berechnung oder so das ist dann immer so $\mathrm{n}$ Punkt ehm den \\
\hline 99 & & einen den konnte man das nur über $\mathrm{n}$ Dreisatz und ganz \\
\hline 600 & & ausführlich und drei Stunden lang Dreisatz erklären und so bis \\
\hline 601 & & sie dann endlich irgendwelche Berechnungen hinkriegten und \\
\hline 602 & & die anderen die ehm die Mathematiker jetzt mal so \\
\hline 603 & Bm: & Waren sofort fertig \\
\hline 604 & Cf: & Ja die waren sofort fertig und die langweilte das entsetzlich \\
\hline 605 & & und die fanden das auch mit dem Dreisatz total doof@und \\
\hline 606 & & meinten@das geht anders viel besser und da merkte man \\
\hline 607 & & wirklich dass diese beiden Gruppen die sassen dann auch noch \\
\hline 608 & & getrennt von $\mathrm{n}$ ander und ehm die dann merkte man wirklich \\
\hline 609 & & dass die da ging irgendwie wie so $\mathrm{n}$ Riss durch den eh durch \\
\hline 610 & & die Gruppe die einen die das ganz ausführlich erklärt haben \\
\hline 611 & & wollten und die anderen die das langweilte und ehm das habe \\
\hline 612 & & ich schon häufiger erlebt dass gerade wenn in diesen \\
\hline 613 & & [...](Gruppe Gipfel) \\
\hline
\end{tabular}

In dieser Passage geht es inhaltlich um die Beschreibung von jenen Schüler/ innen, die mithilfe mathematischer Beschreibungen selbständig und schnell 
Aufgaben im Chemieunterricht lösen. Im Gegensatz zu jenen Schüler/innen, denen das nur mit Unterstützung durch die Lehrerin gelingt, haben sie Mathematik als Leistungskurs neben dem angesprochenen Chemie-LK. Dies führt zu unterrichtlichen Situationen, in denen die «Schnelleren» gelangweilt bzw. unterfordert sind (593-602).

Differenzen, die die Lehrpersonen zwischen den Schüler/innen sehen, sind mit den Möglichkeiten verbunden, Aufgaben in ihrem (naturwissenschaftlichen) Fachunterricht mithilfe mathematischer Lösungswege zu bearbeiten. Über das zur Bewältigung der Aufgabe notwendige Vorwissen verfügt jedoch nur ein Teil des beschriebenen Kurses. Diesen Schüler/innen erklärt die Lehrerin die Berechnungsform (603-613).

$\begin{array}{lll}633 & \text { Am: } & \text { Da hab ich ne ganz gute Erfahrung gemacht und zwar (räuspert } \\ 634 & & \text { sich) hatte ich so ne also so ähnlich aber in Bio hatten wir } \\ 635 & & \text { Vergleich von eh Kurvenverläufen (.) und das stimmt natürlich } \\ 636 & & \text { oder was heist natürlich also die erst mal war beim ersten } \\ 637 & & \text { Mal war es stilles Schweigen und keiner traute sich eh so da } \\ 638 & & \text { ran und dann fingen die ersten an zu beschreiben also die } \\ 639 & & \text { Kurve verläuft so und so die erste ist steiler als die andere } \\ 640 & & \text { und so weiter und so weiter und hatte das dann beschrieben und } \\ 641 & \text { dann stand einer auf und sagte (.) ich mach das mal über } \\ 642 & & \text { (irgen) über Steigungsberechnung und berechnete dann die } \\ 643 & \text { Steigung so und da ging auch hatte ich auch diese Geschichte } \\ 644 & \text { Cf: } & \text { L @(2)@ } \\ 645 & \text { Am: } & \text { ich verstehe überhaupt nichts mehr also du kannst da } \\ 646 & & \text { kannst da meinetwegen noch mehr Steigung berechnen ich weiss } \\ 647 & \text { überhaupt nicht was du da machst und so weiter und dann habe } \\ 648 & \text { ich den Spiess umgedreht und habe ihn sozusagen in der Stunde } \\ 649 & \text { den also spontan den Arbeitsauftrag gegeben dass diejenigen } \\ 650 & \text { die das mathematisch lösen das anderen anders den erklären } \\ 651 & \text { sollen die es mathematisch nicht verstehen und umgekehrt (.) } \\ 652 & \text { mhm also nicht die die ehm also die die das jetzt nicht } \\ 653 & \text { mathematisch lösen aber die sollten sich noch n andern } \\ 654 & \text { Lösungsweg sich überlegen; (.) und das war interessant weil } \\ 655 & \text { dann lösten sich so langsam dieses dieser Riss auf weil die } \\ 656 & \text { diejenigen die das mathematisch lösten merkten wie schwierig } \\ 657 & \text { es ist den anderen das bei zubringen wie man diese Kurve } \\ 658 & \text { beschreibt wie man diese Kurve beschreibt }{ }^{\circ} \text { und wie man }{ }^{\circ} \text { die } \\ 659 & \text { Auswertung macht und }{ }^{\circ} \text { so; }{ }^{\circ} \text { aber das war ne interessante } \\ 660 & \text { Erfahrung weil man dann merkte es wurde so es wurde also die } \\ 661 & \text { Arbeitshaltung wurde viel konzentrierter weil die sich } \\ 662 & \text { natürlich auf zwei Ebenen bewegten nämlich einmal einen System } \\ 663 & \text { zu finden den anderen das beizubringen und [... (Gruppe } \\ 664 & \text { Gipfel) }\end{array}$

In diesem zweiten Teil der Passage wird die Orientierung deutlich, den "Riss» (609) zwischen den Schülergruppen, (nicht) selbständig mathematische Lösungswege für die gestellten Aufgaben zu erkennen und produktiv zur Aufgabenbearbeitung zu nutzen. Hingegen wird der propositionale Anteil der Äusserung Cfs, der sich auf die didaktische Bearbeitung der Situation bezieht, 
antithetisch bearbeitet, indem Am eine konträre Lösung vorstellt. Er beschreibt, wie die Gruppe von Schüler/innen mit dem erforderlichen mathematischen Wissen dies der anderen Gruppe erklärt. So werden die unterschiedlichen Kompetenzen innerhalb der Lerngruppe genutzt, um den Kurvenverlauf mathematisch zu erklären (633-664).

Der positive Horizont, auf den die Orientierung zustrebt, liegt darin, dass möglichst alle Schüler/innen im Unterricht die gestellten Aufgaben mithilfe der mathematischen Sprache bearbeiten und lösen können. Hierbei werden sie von den Lehrpersonen unterstützt, die den Unterricht entsprechend situativ anpassen. Dies verweist auf die Vorstellung einer unterrichtlichen Praxis, die an jenen Schüler/innen orientiert ist, die die fachlichen Voraussetzungen aufgrund nicht-mathematischer Leistungskurse selbständig nicht erfüllen können. Hierin wird die Orientierung deutlich, dass alle Schüler/innen über bestimmte, vorgesehene, fachliche Kompetenzen am Ende einer Unterrichtseinheit verfügen sollen. An diese werden sie, so sie nicht bereits über sie verfügen, durch die Lehrpersonen herangeführt. Differenzen zwischen den Schüler/innen sieht die Gruppe Gipfel zwischen jenen, die über das erwartete Wissen verfügen, und denen, die es unterrichtlich erst erwerben. Hierin wird die Überlegung deutlich, alle Schüler/innen auf einen Stand zu bringen, den sie prinzipiell auch erreichen können. Es wird als Aufgabe des Unterrichts verstanden, dass Erklärungen bereitgestellt werden, also Möglichkeiten eröffnet werden, die Ziele zu erreichen. In der Metapher, den "Riss» zwischen den Gruppen «aufzulösen», also zu schliessen, spitzt sich die Vorstellung zu, dass die gesamte Lerngruppe den gleichen Lösungsweg geht und Differenzen nivelliert werden. Auch wird denjenigen, die bereits den Lösungsweg verstanden haben, eine weitere Lerngelegenheit geboten und zwar zu erklären, d.h. sie erhalten Möglichkeiten zur Erweiterung ihrer begrifflichen Ausdrucksmöglichkeiten.

Aus der Passage wird kein expliziter negativer Horizont deutlich, er lässt sich jedoch implizit darin sehen, dass der Unterricht an jenen Schüler/innen ausgerichtet wäre, die bereits über das geforderte Fachwissen verfügen. Die Praktiken der Lehrpersonen orientieren sich an Vorgaben, die ausserhalb des Unterrichts und seinen Gestaltungsmöglichkeiten liegen, in diesem Fall mathematische Bearbeitungsformen. Die Gruppe grenzt sich gegenüber Lösungswegen ab, die diesen fachlichen Erwartungen nicht entsprechen. Sie stellen jenen Massstab dar, an dem Heterogenität gesehen wird. Berechnungen, die von einigen Schüler/ innen als «besser» eingeschätzt werden, sind hingegen nicht erwünscht. Die Gruppe teilt ein Verständnis von fachlicher Heterogenität, die zu überwinden ist, indem die fehlenden Kompetenzen der benachteiligten Gruppe unterrichtlich aufgegriffen wird. Sie akzeptiert die Unterschiede und versucht, sie unterrichtlich zu kompensieren, also Homogenität des fachlichen Wissens der Schüler/innen herzustellen. 


\section{Differenzkonstruktionen im Vergleich}

Die Kontrastierung der rekonstruierten Orientierungen erfolgt zum einen mit dem Ziel der methodologischen Kontrolle der eigenen Standortgebundenheit (Nohl, 2007a, S. 263 ff.). Zum anderen ist sie auf die Bildung von Typen gerichtet, hier mit dem Fokus auf die Herstellung und Bearbeitung von Differenzen, die sich als Gemeinsamkeit über die Fälle und Typen hinweg entlang von Erwartungen an die Rolle der Schüler/innen bzw. Abweichungen von dieser herauskristallisieren. Die Bezugspunkte scheinen ausserhalb des Unterrichts und somit der professionellen Gestaltungsmöglichkeiten der Lehrpersonen zu liegen, nämlich in der schulisch-administrativen, ressourcenzuweisenden Kategorie des sonderpädagogischen Förderbedarfs (Kontinent) und in den zu erreichenden Lernzielen (Gipfel). Für die Gruppe Kontinent liegt die Erwartung in dem Potenzial der Schüler/innen, aus eigener Kraft oder mittels gesellschaftlicher ausserschulischer Kompensations- und Unterstützungsleistungen dem Unterricht folgen zu können. Die Lehrpersonen unterscheiden dabei zwischen Erwartungen an die Regelschul- und an die Integrationskinder, die sie dichotom und total beschreiben, d.h. die Schüler/innen werden der einen oder der anderen Gruppe zugeordnet. Die Gruppe Gipfel hingegen erwartet das Erreichen inhaltlicher Vorgaben, an die sie die Schüler/innen heranführt. Anders als die Gruppe Kontinent bezieht sich die Gruppe Gipfel auf Teilaspekte der Schüler/innen, die sie in der Logik fachbezogener Erwartungen und Abweichungen im Unterricht konstruiert.

Hinsichtlich der unterrichtlichen Orientierungen der Differenzbearbeitung unterscheiden sich die illustrierten Typen. Die Gruppe Gipfel nimmt die fachbezogene Differenz als Ausgangspunkt für ihre unterrichtliche Gestaltung mit dem Ziel, die fachlichen Niveaus der Schüler/innen einander anzugleichen. Hierin findet sich die Orientierung, eine homogene Lerngruppe bezüglich der fachlichen Leistungen zu erreichen. Darin ist die Möglichkeit enthalten, dass sie die «Defizite» als überwindbar ansieht. Im Gegensatz dazu verortet die Gruppe Kontinent die Handlungsoptionen zur Bearbeitung von einer heterogenen Lerngruppe ausserhalb der Schule und des Unterrichts; gleichermassen orientiert an der Vorstellung, Homogenität zu erreichen. In ihrer unterrichtlichen Praxis führt dies jedoch - anders als in der Gruppe Gipfel - zur Akzeptanz der bestehenden heterogenen Lerngruppe.

In beiden Typen wird die Orientierung an einem ausserunterrichtlichen Massstab auf formale Regeln der Organisation Schule deutlich, mit denen sie im Einklang stehen und/oder sich zugleich widersprechen. Die schulische Regelung des Vergleichs von Schüler/innen untereinander sowie in Relation zu diesen formalen Regeln findet hier ihren Ausdruck. In Typ 2, repräsentiert durch die Gruppe Kontinent, wird diese Erwartung als unterrichtlicher Bezugspunkt genommen und die Vorstellungen harmonisieren entsprechend mit der formalen Regel der Besser-/Schlechterstellung der Schule. Dabei reproduzieren sie soziale Unterschiede zwischen Schüler/innen. Die durch den Fall der Gruppe Gipfel 
illustrierten Erwartungen harmonisieren insofern mit der aufgezeigten Regel, als dass die Schüler/innen an die gegebenen Ziele herangeführt und dabei verglichen werden. Dabei kommt dieser Typ zugleich dem schulischen Anspruch nach, Unterschiede zwischen Schüler/innen auszugleichen. Die formale Regel, für alle Schüler/innen Lerngelegenheiten zu schaffen, wird ebenfalls erfüllt, wenn auch widersprüchlich. Schüler/innen, die bereits über das erwartete Wissen verfügen, erhalten die Möglichkeit, es zu vertiefen, indem sie es ihren Mitschüler/innen erklären. Die im Widerspruch zueinander stehenden formalen Regeln werden beide befolgt, indem allen soziale, nicht jedoch inhaltliche Lernmöglichkeiten eröffnet werden. Dies stellt insofern eine Behinderung des fachlichen Lernens dar, als dass jene Schüler/innen, die über die erwarteten mathematischen Kompetenzen bereits verfügen, weniger Gelegenheiten zum Lernen fachbezogener Inhalte erhalten. In dem von der Gruppe Kontinent repräsentierten Typ sind es die "Kinder aus dem Niemandsland», die keine an ihre Lernsituation angepassten unterrichtlichen Angebote erhalten. Anders als in Typ 2 werden in Typ 4 keine anderen Lerngelegenheiten eröffnet, wie im Bereich des Erklärens.

\section{Implizite und explizite unterrichtliche Differenzkonstruktionen reflektieren}

Die Ausführungen zeigen, dass Konstruktion und Bearbeitung von Differenzen im Unterricht, also der Heterogenität der Schüler/innen, durch komplexe Praxen gekennzeichnet ist, die im schulorganisatorischen Rahmen eingebunden sind. Die Orientierungen sind nicht für sich zu betrachten, sondern als Ausdruck eines Milieus, das sich im Kontext der Organisation Schule, die ihrerseits Teil der Gesellschaft ist, entwickelt (hat). So konnte gezeigt werden, dass die Differenzkonstruktionen der Lehrpersonen im Zusammenhang mit schulisch-formalen Regeln stehen. Die Anforderungen, die mit der Lehrerrolle in Schule einhergehen, als Grundlage für Selektionsentscheide im Modus besser/schlechter zu bewerten und zugleich Lern- und Bildungsprozesse zu initiieren, findet sich in den expliziten und impliziten Differenzkonstruktionen der Lehrpersonen. Dies verbindet sich mit ihren unterrichtlichen Praktiken, die daran orientiert sind, Differenzen zu reproduzieren und auszubauen bzw. sie zugunsten homogener Lernstände anzugleichen. In ihrem Zusammenspiel führt dies zu spezifischen schulischen Differenzformen im Bereich schulischer Leistung. Überlegungen, wie ein veränderter Umgang mit Heterogenität in Schule und Unterricht aussehen könnte, sollten diese Komplexität unterrichtlicher Differenzkonstruktionen nicht ausblenden, sondern den «von Differenzen präformierten Kontext» (Mecheril \& Plösser, 2009, S. 206) der Schule reflexiv einbeziehen. Mögliche Anknüpfungspunkte für einen Unterricht, der Marginalisierungen überwindet, also Ansätze einer konstruktiven und kooperativen Unterrichtspraxis, sind im Typ 2, illustriert am Beispiel der Gruppe Gipfel, insofern vorhanden, als dass 
unterschiedliche Lernziele innerhalb einer Klasse als möglich angesehen und im gemeinsamen Unterricht bearbeitet werden können. Eine solche Perspektive über das Ziel der (situativ auftauchenden) Kompensation des Nicht-Wissens einer Teilgruppe von Schüler/innen hinausgehend anzuwenden, bietet Möglichkeiten der Weiterentwicklung einer integrations- respektive inklusionsfähigen Schule (Bless \& Kronig, 1999). Unterricht, in dem weniger Benachteiligungen wirksam werden, scheint darauf angewiesen, das widersprüchliche Spannungsgeflecht, in dem die unterrichtlichen Praktiken eingebettet sind, zu reflektieren. Zugleich untermauern die Erkenntnisse eine Vorstellung von Inklusion als unter den gegebenen schulischen und gesellschaftlichen Rahmenbedingungen - «not a final destination but something that must be struggled for every day and by everyone» (Allan, 2012).

Die Ergebnisse zeigen, dass die impliziten Formen der Herstellung und Bearbeitung von Heterogenität im professionellen Lehrermilieu in Zusammenhang mit dem formal-expliziten und widersprüchlichen Verhältnis von Bildungs- und Selektionsfunktion der Schule stehen, das von den Lehrkräften in Erwartungen an die Schülerrolle übersetzt wird. Unterrichtliche Entwicklung zugunsten einer inklusiveren Praxis bedarf der Anerkennung eines praxisbezogenen Unterrichtswissens, das in seiner Relation zu den expliziten Vorgaben zu Differenz reflektiert wird. Inwiefern die rekonstruierten Typen einem professionellen Selbstverständnis von Lehrpersonen in unterschiedlichen Stufen selektiver und terminaler Schulsysteme entsprechen, in denen Grundschule und Gymnasien unterschiedliche Rollen einnehmen, wäre durch Kontrastierungen mit weniger selektiven Systemen empirisch zu überprüfen.

\section{Anmerkungen}

1 Alle Namen der Diskutierenden sind maskiert, der Grossbuchstabe steht für eine Person, das $\mathrm{f}$ für eine Frau und $\mathrm{m}$ für einen Mann. Die Namen der Gruppen sind frei erfunden. Die Zeilennummern entsprechen denen des Originaltranskripts. Die Transkription orientiert sich an den Regeln der TiQ, Transcript in qualitative research (Przyborski \& Wohlrab-Sahr 2009, S. 166 ff.). Das «@»-Zeichen verweist im Transkript auf Lachen.

2 Integrativ bezieht sich hier auf die gemeinsame Beschulung von Kindern mit und ohne sonderpädagogischen Förderbedarf.

\section{Literatur}

Allan, J. (2012). Inclusion: Patterns and Possibilities. Zeitschrift für Inklusion, 4. Zugriff am 25.01.2013 unter http://www.inklusion-online.net/index.php/inklusion/article/ view/183/171

Ainscow, M. (2008). Teaching for diversity. The Next Big Challange. In: F. M. Connelly et al. (Ed.), The Sage Handbook of Curriculum and Instruction (pp. 240-258). Los Angeles, CA: SAGE Publications.

Bless, G. \& Kronig, W. (1999). Wie integrationsfähig ist die Schweizer Schule geworden? Eine bildungspolitische Analyse über schulorganisatorische Massnahmen bei Normabweichungen. Vierteljahreszeitschrift für Heilpädagogik und ihre Nachbargebiete, 68 (4), 414-426. 
Bohnsack, R. (2010). Rekonstruktive Sozialforschung. Einführung in qualitative Methoden (8., durchges. Aufl.). Opladen \& Farmington Hills, MI: Verlag Barbara Budrich.

Bohnsack, R. \& Nohl, A.-M. (2001). Ethnisierung und Differenzerfahrung. Zeitschrift für Qualitative Bildungs-, Beratungs- und Sozialforschung, 1, 15-36.

Budde, J. (2012). Die Rede von der Heterogenität in der Schulpädagogik. Diskursanalytische Perspektiven [63 Absätze]. Forum Qualitative Sozialforschung / Forum Qualitative Social Research, 13 (2), Art. 16. Zugriff am 09.05.2012 unter http://nbn-resolving.de/urn:nbn:de:0114-fqs1202160

Garfinkel, H. (1956). Conditions of Successful Degregation Ceremonies. American Journal of Sociology, 61 (5), 420-424.

Mannheim, K. (1980). Strukturen des Denkens. Frankfurt/Main: Suhrkamp.

Mecheril, P. \& Plösser, M. (2009). Differenz und Pädagogik. In S. Andresen et al. (Hrsg.), Handwörterbuch Erziehungswissenschaft (S. 194-208). Weinheim: Beltz.

Nohl, A.-M. (2007a). Komparative Analyse: Forschungspraxis und Methodologie dokumentarischer Interpretation. In R. Bohnsack et al. (Hrsg.), Die dokumentarische Methode und ihre Forschungspraxis. Grundlagen qualitativer Sozialforschung (S. 255-276). Wiesbaden: VS Verlag für Sozialwissenschaften.

Nohl, A.-M. (2007b). Kulturelle Vielfalt als Herausforderung für pädagogische Organisationen. Zeitschrift für Erziehungswissenschaft, 10 (1), 61-74.

Nohl, A.-M. (2010). Konzepte interkultureller Pädagogik. Eine systematische Einführung (2., erw. Aufl.). Bad Heilbrunn: Verlag Julius Klinkhardt.

Prengel, A.(2006). Pädagogik der Vielfalt. Verschiedenheit und Gleichberechtigung in Interkultureller, Feministischer und Integrativer Pädagogik (3. Aufl.). Wiesbaden: VS Verlag für Sozialwissenschaften.

Przyborski, A. \& Wohlrab-Sahr, M. (2009). Qualitative Sozialforschung (2., korrig. Aufl.). München: Oldenbourg Verlag.

Sturm, T. (2010). Schulentwicklung: Exklusionsrisiken und Inklusionspotenziale. In N. Wenning et al. (Hrsg.), Strategien der Ausgrenzung - Exkludierende Effekte staatlicher Politik und alltäglicher Praktiken in Bildung und Gesellschaft (S. 131-150). Münster: Waxmann.

Sturm, T. (2012). Praxeologische Unterrichtsforschung und ihr Beitrag zu inklusivem Unterricht. In Inklusion online 1. Zugriff am 28.05.2013 unter http://www.inklusion-online. net/index.php/inklusion/article/view/151/143

Wagner-Willi, M. \& Sturm, T. (2012). Inklusion und Milieus in schulischen Organisationen. Inklusion online, 4. Zugriff am 28.01.2013 unter http://www.inklusion-online.net/index. $\mathrm{php/inklusion/article/view/185/173}$

Zahner Rossier, C. \& Holzer, T. (2007). PISA 2006: Kompetenzen für das Leben - Schwerpunkt Naturwissenschaften. Nationaler Bericht. Neuchâtel: Bundesamt für Statistik.

Schlagworte: Heterogenität, Differenz, Inklusion, Unterricht, dokumentarische Methode 


\section{La (re-)production des différences entre élèves dans différents styles d'enseignement}

\section{Résumé}

Cette contribution examine, sous l'angle de la sociologie des connaissances, comment les enseignants produisent et gèrent des différences entre élèves au cours de leurs pratiques d'enseignement. À partir d'un matériau empirique constitué de discussions en groupe, deux styles d'enseignement sont identifiés: le premier comprend des pratiques totalement centrées sur l'atteinte d'une norme, tandis que le second concerne des pratiques plus adaptatives aux composantes situationnelles. Les deux styles peuvent entraver et faciliter les opportunités d'apprentissage en classe. En outre, les résultats confirment l'importance des règles scolaires formelles dans les pratiques d'enseignement. Ces constats doivent être pris en compte dans la mise en place d'une école et d'un enseignement inclusifs.

Mots-clés: Hétérogénéité, reproduction de la différence, inclusion, style d'enseignement, méthode d'analyse documentaire

\section{(Ri)Produzione delle differenze negli orientamenti d'insegnamento}

\section{Riassunto}

Parallelamente a un approccio tipico della sociologia del sapere, nel saggio viene approfondita la domanda di come i docenti creino ed elaborino differenze tra scolare e scolari nella pratica didattica. Sulla base di materiale empirico derivante da discussioni in gruppo vengono illustrate due tipologie differenti: una orientata a una norma totale, l'altra che tende ad adeguarsi alle situazioni. Entrambe le forme ostacolano e aprono opportunità d'insegnamento durante la lezione. Inoltre, forniscono indizi di come venga data grande importanza alle regole scolastiche formali nelle pratiche d'insegnamento dei docenti. Nella strutturazione di una pratica d'insegnamento e scolastica inclusiva occorre tenere conto di questi punti d'orientamento.

Parole chiave: Eterogenietà, differenza, inclusion, insegnamento, metodo documentario 


\title{
Re)Production of differences in different teaching orientations
}

\author{
Summary \\ With respect to a sociology of knowledge theoretical framework, this article \\ investigates how teachers construct and deal with differences among students \\ in their teaching practices. Discursive empirical data generated within focus \\ groups enable to identify two teaching orientations: one consists of normative \\ practices, while the other concerns more situation-related adaptive practices. \\ Both teaching orientations hinder and foster students' learning opportunities. \\ Moreover, results emphasize the importance to consider formal school rules \\ within teaching practices. These aspects need to be taken into account when \\ shaping inclusive schools and teaching.
}

Keywords: Heterogeneity, difference reproduction, inclusion, teaching orientation, documentary method 